\title{
L'espace dans Madame Bovary
}

Paula Roberts

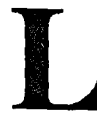

a valeur du travail de Flaubert est telle que cet auteur demeure un des plus étudiés du monde; innovatrice et pure, son expression est lourde de signification. A. Cook commente que chaque détail a une fonction double: comme un détail en soi mais également comme un reflet de l'action.' La description, les arrière-plans, les objets: tout élément est doublement significatif.

Dans Madame Bovary, l'espace joue un rôle intégral dans le déroulement du récit; il reflète et explique les personnages. En dépit du fait que le roman entier se déroule dans la Normandie, il existe diverses divisions spatiales qui revêtent une valeur symbolique. Qui plus est, les déplacements des personnages et les changements et métamorphoses spatiaux appuient et amplifient certains thèmes intégraux.

La campagne rouennaise, "contrée bâtarde où le langage est sans accentuation, comme le paysage sans caractère," 2 est plate et tranquille, sans contours abrupts; c'est l'antithèse du caractère capricieux et sybarite d'Emma. Elle s'ennuie dans cet espace, fixant constamment l'horizon et cherchant dans l'au-dela les voluptés du rêve:

Plus les choses, d'ailleurs, étaient voisines, plus sa pensée s'en détournait. Tout ce qui l'entourait immédiatement, campagne ennuyeuse, petits bourgeois imbéciles, médiocrité de l'existence, lui semblait une exception dans le monde, un hasard particulier où elle se trouvait prise, tandis qu'au-delà s'étendait à perte de vue l'immense pays des félicités et des passions. (379)

Comme l'observe Poulet:

La pensée d'Emma est d'abord toute périphérique. Elle se promène à l'horizon, vagabonde de droite et de gauche, erre dans les lointains qui sont à la fois ceux du paysage extérieur où se perd le regard, et ceux du paysage interne où s'égare 


\section{rêverie. $^{3}$}

Emma croit que son espace à elle est une erreur du hasard; la vie qu'elle devrait mener est ailleurs. Cette illusion informe son goût pour Paris et pour des pays exotiques:

Comment était ce Paris? Quel nom démesuré! Elle se le répétait à demi-voix, pour se faire plaisir; il sonnait à ses oreilles comme un bourdon de cathédrale, il flamboyait à ses yeux jusque sur l'étiquette de ses pots de pommade. (377)

Charles, par contre, est tout satisfait de l'ici et du présent: "il possédait pour la vie cette jolie femme qu'il adorait. L'univers, pour lui, n'excédait pas le tour soyeux de son jupon...." (356) L'opposition fondamentale entre les deux personnages et leurs attitudes envers la vie est soulignée par la conception que chacun fait de son environnement.

La vie à Tostes n'est qu'une ébauche de la vie future à Yonville. Pendant cette étape initiale, l'espace est limité aux endroits fréquentés par Charles et Emma; il n'y a que les personnalités de ceux-ci qui sont développées. C'est l'époque de la vie à deux et de la fidélité. L'isolement des personnages dans l'espace permet au lecteur de mieux les saisir et permet à Emma d'apprendre le décalage entre ses rêves et la réalité.

Les autres personnages entrent en jeu lors du déménagement à Yonville. En quittant Tostes, on abandonne la vie conjugale: le dernier geste d'Emma avant leur départ est de jeter au feu son bouquet de mariage. L'introduction des autres personnages dans le nouvel espace d'Yonville signifie la dissolution du couple et le commencement d'une existence différente, à savoir l'engloutissement d'Emma.

A Yonville, chaque maison incarne le personnage qui y demeure. Chez Homais, c'est une atmosphère de confort coutumier. Chaque objet a une place et une fonction; Homais administre son domaine comme il dominerait la société. La toile cirée sur la table, les grilles de fer sur les fenêtres, tout témoigne du bon sens bourgeois, de la fatuité et de l'esprit canonique du pharmacien.

Le capharnaüm de Homais est le lieu inviolable de la Science: "Personne au monde n'y mettait les pieds; et il le respectait si fort, qu'il le balayait lui-même." (551) Cet endroit privé représente l'égoïsme fondamental du caractère bourgeois. La pharmacie, par contre, reflète son influence, son orgueil et son indiscrétion:

Mais ce qui attire le plus les yeux,c'est,en face de l'auberge du Lion d'or, la pharmacie de M. Homais!... l'enseigne, qui tient toute la largeur de la boutique, porte en lettres d'or: Homais, pharmacien. Puis, au fond de la boutique, derrière les grandes balances scellées sur le comptoir, le mot laboratoire se déroule, au-dessus d'une porte vitrée, qui, à moitié de sa hauteur, répète encore une fois Homais, en lettres d'or....(390-1)

Les enseignes brillantes créent un mythe qui dépasse l'homme, ainsi que son pouvoir éclipse sa bêtise. Les deux côtés de la personnalité du pharmacien sont donc bien représentés dans la démarcation spatiale entre la pharmacie et le capharnaüm.

Guillaumin, le notaire, vit dans une opulence redondante et fière. Il se permet 
le luxe d'un poêle en porcélaine, pareil à celui de la Vaubyessard. Sa collection de meubles et de bibelots est moins une attestation de son goût personnel que d'un train de vie plutôt élevé. Dans le jardin, il y a une statue de Cupide, et dans la salle à manger:

il y avait la Esmérelda de Steuben, avec la Putiphar de Schopin. La table servie, deux rechauds d'argent, le bouton des portes en cristal, le parquet et les meubles, tout reluisait d'une propreté méticuleuse, anglaise.... (600)

Le décor de cette maison exprime la convoitise du propriétaire; le notaire aimerait acheter Emma comme il acheterait ce mobilier.

Bournisien, nul et négligé, ressemble à son église. Celle-ci:

a été rebâtie à neuf dans les dernières années du règne de

Charles X. La voûte en bois commence à se pourrir par le haut, et, de place en place, a des enfonéures noires dans sa couleur bleue. (390)

La représentation négative de l'église et du curé est un commentaire oblique sur la condition de la religion au dix-neuvième siècle.

Le nom de la demeure de Rodolphe, "La Huchette" ("boîte pour pétrir la pain") va de pair avec son nom de famille (Boulanger). L'atmosphère ici revêt une aisance nonchalante: les pièces sont ornées d'objets pompeux qui fascinent puis dégoûtent Emma. Elle rage:

Mais, lorsqu'on est si pauvre, on ne met pas d'argent à la crosse de son fusil! On n'achète pas une pendule avec des incrustations d'écaille! continuait-elle en montrant l'horloge de Boulle; ni des sifflets de vermeil pour ses fouets -elle les touchait!- ni des breloques pour sa montre! (610)

Pareil à ces objets, Rodolphe lui aussi est doré: sa médiocrité est dissimulée sous un panache superficiel. Quand il écrit la lettre fatale à Emma, il est assis audessous d'une tête de cerf, c'est-à-dire sous le signe de la bêtise. Comme Rodolphe, la maison est pleine d'éclat mais sans authenticité.

La maison de Charles et Emma n'est pas moins symbolique; il y a un motif recurrent de l'étroitesse. Aux approches d'Yonville, "les cours se font plus étroites, les habitations se rapprochent, les haies disparaissent." (389) Le village entier a un air étriqué, mais la maison du docteur en est le microcosme. Le plafond est trop bas et Emma se lamente "du bonheur qui lui manquait, de ses rêves trop hauts, de sa maison trop étroite." (424) Dans une même phrase, Emma combine l'espace et le sentiment: sa maison l'étouffe physiquement et émotionnellement; elle doit souvent aller à la fenêtre pour respirer et apaiser le tourbillon de ses pensées.

Cette fenêtre représente simultanément l'ouverture et la clôture, la fuite et la barrière. Emma s'y accoude régulièrement: "Elle s'y mettait souvent: la fenêtre, en province, remplace les thêâtres et la promenade." (441) Encore une fois, l'espace renferme la dichotomie entre le rêve (théâtre, promenade) et la réalité (fenêtre).

C'est par la fenêtre qu'Emma regarde le départ de Charles chaque matin, quand elle a toujours de grandes expectations pour le mariage: 
Elle se mettait à la fenêtre pour le voir partir; et elle restait accoudée sur le bord, entre deux pots de géraniums, vêtue de son peignoir...et elle continuait à lui parler d'en haut, tout en arrachant avec sa bouche quelque bribe de fleur ou de verdure qu'elle soufflait vers lui.... (355)

Plus tard, elle guette l'ombre de Léon qui passe par la fenêtre de leur maison chaque soir, et elle est à la fenêtre lorsqu'elle aperçoit Rodolphe pour la première fois.

Néanmoins, la fenêtre représente la frustration aussi bien que la rêverie. Elle encadre le départ de Léon qui quitte Yonville pour Paris. Après avoir reçu la nouvelle que Rodolphe la quitte, Emma voit son tilbury bleu passer par la place, sous la fenêtre.

Pour Emma, la fenêtre est un moyen de se distancer de la réalité: Emma est à l'intérieur et elle promène ses yeux sur les scènes extérieures du village comme si elle n'y appartient pas. Elle se détache du spectacle qui joue sous ses yeux et se laisse emporter par son imagination:

[B]rûlée plus forte par cette flamme intime que l'adultère avivait, haletante, émue, tout en désir, elle ouvrait sa fenêtre, aspirait l'air froid, éparpillait au vent sa chevelure trop lourde, et, regardant les étoiles, souhaitait des amours de prince. (588-9)

Donc, la fenêtre est à la fois une ouverture vers le rêve et un cadre pour les frustrations d'Emma.

Si le présent temporel n'est pas acceptable pour Emma, l'ici de l'espace ne l'est guère plus. Ce sont les espaces du rêve ou du passé qui préoccupent l'héroïne. Elle rappelle son couvent avec nostalgie parce que cet endroit, pareil à une prison, avait bloqué la réalité de la vie extérieure. Pendant ces années d'isolement, Emma avait appris à juger l'existence par les livres qu'elle lisait clandestinement, donc:

Elle n'aimait la mer qu'à cause de ses tempêtes, et la verdure seulement lorsqu'elle était clarsemée parmi les ruines. Il fallait qu'elle pût retirer des choses une sorte de profit personnel; et elle rejetait comme inutile tout ce qui ne contribuait pas à la consommation immédiate de son coeur, -étant de tempérament plus sentimentale qu'artiste, cherchant des émotions et non des paysages. (358)

La dernière phrase capte en microcosme la dichotomie entre espace de rêve et espace réel dans la pensée d'Emma. L'ennui commence dès qu'elle quitte le couvent:

Emma, rentrée chez elle, se plut d'abord au commandement des domestiques, prit ensuite la campagne en dégoût et regretta son couvent. Quand Charles vint aux Bertaux pour la première fois, elle se considérait comme fort désillusionnée, n'ayant plus rien à apprendre, ne devant plus rien sentir. (361)

Emma ne cesse jamais de regretter les rêves naïfs de sa jeunesse. Après avoir goûté de la vie conjugale et de deux liaisons adultères, Emma préfère toujours 
l'espace protégé et irréaliste du couvent. Après une rencontre gâtée avec Léon. elle s'assoit contre le mur du couvent et songe:

Quel calme dans ce temps-là comme elle enviait les ineffables sentiments d'amour qu'elle tâchait, d'après les livres, de se figurer!...D'où venait donc cette insuffisance de la vie, cette pourriture instantanée des choses où elle s'appuyait?...tout mentait! (584)

En fait, le couvent est une mise en abyme du roman entier: l'ennui mène aux rêves, puis à un désir de s'échapper, ensuite à la confusion et le désenchantement, et le cycle recommence avec l'ennui.

L'espace de la Vaubyessard correspond aussi au regret du passé et aux expectations pour l'avenir. Au château, Emma éprouve pour la première fois la volupté du luxe et de l'exotisme; c'est cet espace qui concrétise ses rêves de jeune fille. Le raffinement de l'ambiance, les plats exquis et exotiques, la société aristocrate. tout s'oppose diamétralement à la plus grande et la plus récente fête de la vie d'Emma: son mariage. Elle est séduite par ce luxe; la haute société la fascine, et elle est très sensible à l'abîme entre sa vie à elle et l'atmosphère enivrante du château. Emma voit des paysans qui regardent le bal du dehors:

Alors, le souvenir des Bertaux lui arriva. Elle revit la ferme, la mare bourbeuse, son père en blouse sous les pommiers, et elle se revit elle-même, comme autrefois, écrémant avec son doigt les terrines de lait dans la laiterie. Mais, aux fulgurations de l'heure présente, sa vie passée, si nette jusqu'alors,s'évanouissait tout entière, et elle doutait presque de l'avoir vécue. (372)

Emma adopte le monde de la Vaubyessard comme son milieu prédestiné; c'est ici que nât son bovarysme, son désir d'être autre qu'elle ne l'est. Léon et Rodolphe ne seront que des projections du Vicomte avec qui elle valse. Cet espace agit sur elle et détermine l'acheminement de ses désirs:

Le souvenir du Vicomte revenait toujours dans ses lectures. Entre lui et les personnages inventés, elle établissait des rapprochements. Mais le cercle dont il était le centre peu à peu s'élargit autour de lui, et cette auréole qu'il avait, s'écartant de sa figure, s'étala plus au loin, pour illuminer d'autres rêves. (378)

Ce n'est donc pas par hasard que le Vicomte et le couvent reparaissent à l'époque de la chute d'Emma $(584,597)$ car ils lui signalent l'echec de tous ses espoirs. Ces deux espaces sont d'abord des amorces pour les rêves de l'avenir. mais plus tard ils sont des sommets passés qui ne verront rien de comparable.

Les voyages et les mouvements dans l'espace sont significatifs aussi. $\mathrm{La}$ valeur connotative des actions transcende leur simple rôle diégétique. Les déplacements de Charles et Emma sont antithétiques: le médecin parcourt la campagne pour soigner des malades et pour gagner de l'argent; ses voyages sont humanitaires et utiles. Emma, par contre, part pour dépenser cet argent, pour s'indulger et pour poursuivre ses rêves. Elle va à la Huchette pour être avec Rodolphe et à Rouen pour ses liaisons avec Léon. De plus, ces excursions se basent sur le mensonge: Emma manipule Charles pour qu'elle puisse passer du 
temps avec Léon; c'est sous le prétexte de "leçons de piano" qu'elle va à Rouen. Donc, les motivations derrière les déplacements des deux personnages reflètent leurs personnalités antithétiques.

La vitesse de ces mouvements est un autre aspect important. Charles se lie surtout à la lenteur: il travaille comme un "cheval de manège", et le nom "Bovary" suggère la rumination. Chez Emma, la rapidité est employée pour traduire le délire de ses passions. Pendant sa valse à la Vaubyessard, le rythme accélère progressivement et à la fin de la danse Emma est défaillante:

haletante, elle faillit tomber, et, un instant, s'appuya la tête sur sa poitrine. Et puis, tournant toujours, mais plus doucement, il la réconduisit à sa place; elle se renversa contre la muraille et mit sa main devant ses yeux. (373)

C'est une métaphore de l'acte sexuel qui reparaît d'ailleurs dans le galop des chevaux avant qu'elle se donne a Rodolphe et dans la course folle du fiacre dans lequel elle se donne à Léon:

De temps à autre, le cocher, sur son siège, jetait aux cabarets des regards désepérés. Il ne comprenait pas quelle fureur de la locomotion poussait ces individus à ne vouloir point s'arrêter. Il essayait quelquefois, et aussitôt il entendait derrière lui partir des exclamations de colère. Alors il cinglait de plus belle ses deux rosses tout en sueur.... (549)

Emma subit souvent des sensations de "tourbillon" ou d"'oscillation": "Il lui semblait que le sol de la place oscillante s'élevait le long des murs, et que le plancher s'inclinait par le bout, à la manière d'un vaisseau qui tangue." (513) Sa violence émotive est reflétée par la turbulence dans l'espace environnant tout comme l'ennui s'associe au paysage plat et étroit. Selon M. Bal la description flaubertienne renferme souvent "une alternance entre le mouvement et l'immobilité, l'espace et le rétrécissement, le progrès et la stagnation, analogue à l'oscillation existant à l'intérieur du personnage d'Emma." 4

A ces mouvements et déplacements se combine un jeu de montée et descente qui incarne l'état d'âme de l'héroïne. Emma est souvent "en haut", ce qui représente l'ampleur de ses aspirations, la prédominance de la passion et même son isolement. Sa chambre est en haut; quand Charles devient absolument insoutenable, il est exilé:

Madame était dans sa chambre. On n'y montait pas. Elle restait là tout le long du jour, engourdie, à peine vêtue...Pour ne pas avoir, la nuit, auprès d'elle, cet homme étendu qui dormait, elle finit, à force de grimaces, par le reléguer au second étage.... (588)

En chassant Charles de sa chambre elle bannit en même temps la réalité.

Aux Comices, Emma et Rodolphe sont d'abord au même niveau que le reste du monde, mais ils montent bientôt à la mairie pour surveiller de loin les événements. Leur intégration initiale dans la foule symbolise leur appartenance à cet espace et à ce groupe, malgré le mépris d'Emma: "Et, tout en se moquant des comices, Rodolphe, pour circuler plus à l'aise, montrait au gendarme sa pancarte bleue, et même il s'arrêtait parfois devant quelque beau sujet, que Mme 
Bovary n'admirait guère." (451) La montée à la mairie signifie le désir d'Emma de s'éloigner de son milieu naturel et de se mettre au-dessus de la réalité. Cependant, ce recul reste temporaire.

Aux Comices, le morcellement spatial correspond à une échelle sociale: au plus bas se trouvent le bétail et les paysans, plus haut sont les bourgeois du village, sur l'estrade est M. le Conseiller et son entourage, et Emma et Rodolphe sont encore plus élevés, dans l'espace des illusions. Cependant, tous ces niveaux sont décrits concurremment; la description saute indifféremment entre eux, et la bêtise transcendante enveloppe tous, y compris Emma.

L'élévation dans l'espace traduit souvent la fièvre de l'émotion ou de la passion: après avoir reçu la lettre de Rodolphe, Emma court au grenier et contemple se suicider:

Elle s'était appuyée contre l'embrasure de la mansarde et elle relisait la lettre avec des ricanements de colère. Mais plus elle y fixait d'attention, plus ses idées se confondaient...Elle jetait les yeux tout autour d'elle avec l'envie que la terre croulît. Pourquoi n'en pas finir? Qui la retenait donc? Elle était libre. Et elle s'avança, elle regarda les pavés.... (513)

Pareillement, dans la forêt avec Rodolphe, elle surveille Yonville au-dessous d'elle: "jamais ce pauvre village où elle vivait ne lui avait semblé si petit." (46970) A l'Opéra, quand elle s'éprend de Lagardy et rencontre Léon, elle est en haut, dans les "premières". Quand elle se donne à Léon, le fiacre est au-dessus de Rouen. Donc, l'élévation physique correspond souvent aux sommets émotionnels dans la vie d'Emma. ${ }^{\text {s }}$

La diligence (L'Hirondelle) symbolise l'alternance entre le rêve et la réalité. Chaque jeudi, Emma part vers le rêve (Rouen) seulement pour rentrer plus tard à la réalité (Yonville). Le voyage de retour est une descente à la fois physique et émotionnelle: "Cela lui descendait au fond de l'âme comme un tourbillon dans un abîme, et l'emportait parmi les espaces d'une mélancolie sans bornes." (569) D'ailleurs, c'est seulement pendant les voyages de retour que se montre l'Aveugle, symbole de la Némésis et de la mauvaise conscience d'Emma.

Les métamorphoses dans l'espace fonctionnent fréquemment comme un miroir des sentiments de l'héroïne. Le ciel, par exemple, extériorise l'âme d'Emma. Pendant ses promenades à Tostes, le ciel morne, monotone et gris reflète son ennui. Pendant une promenade avec Rodolphe, par contre, le ciel est bleu, et il fait un temps parfait lorsque Léon l'accompagne chez la nourrice.

Les étoiles et la lune ne figurent dans la description que quand Emma songe ou quand elle rencontre un amant, surtout lors du rendez-vous avec Rodolphe dans le jardin; à ce moment-là , Emma est au comble de bonheur, croyant qu'on partira ensemble pour réaliser ses rêves:

La lune, tout ronde et couleur de pourpre, se levait à ras de terre, au fond de la prairie...Puis elle parut, éclatante de blancheur, dans le ciel vide qu'elle éclairait; et alors, se ralentissant, elle laissa tomber sur la rivière une grande tache, qui faisait une infinité d'étoiles; et cette lueur d'argent semblait s'y tordre jusqu'au fond à la manière d'un serpent sans tête couvert d'écailles lumineuses. (506) 
Alors, les différentes sortes de lumière qui jouent comme arrière-plan fonctionnent également comme baromètre des émotions d'Emma; la lumière est plus manifeste (bien qu'éphémère) lorsqu'Emma se croit contente; mais d'habitude l'aspect gris et morne domine le paysage comme il domine l'existence. Auerbach affirme:

A force de tableaux qui transforment le néant de la vie quotidienne en un état oppressant de dégoût et d'ennui, où se mêlent les espoirs fallacieux, les déceptions paralysantes et les craintes pitoyables, nous voyons une grise et banale destinée humaine s'acheminer lentement vers sa fin. ${ }^{6}$

Les couchers de soleil, par contre, correspondent souvent aux moments de chagrin. Lors d'une entrevue ratée entre Emma et Bournisien, ce dernier est décrit ainsi: "La lueur du soleil couchant qui frappait en plein son visage pâlissait le lasting de sa soutane, luisante sous les coudes, effiloquée par le bas." (427) Alors dans les deux sens le Ciel offre peu de lumière à Emma. Un autre coucher de soleil encadre le départ du père Rouault après les funerailles de sa fille: il s'agit de la fin définitive des rêves.

Ironiquement, pendant l'enterrement d'Emma il fait un temps parfait; tout semble se moquer de cette vie manquée:

Une brise fraîche soufflait, les seigles et les colzas verdoyaient, des gouttelettes de rosée tremblaient au bord du chemin, sur les haies d'épines...Le ciel pur était tacheté de nuages roses; des lumignons bleuâtres se rabattaient sur les chaumières couvertes d'iris.... (634)

En plus, il y a un réseau d'images fluides qui fait parallèle à la dissolution humaine continue dans le roman. Au dire de V. Brombert, la vie selon Flaubert est un processus continu de déchéance. ${ }^{7}$ L'eau, les vapeurs et les nuages figurent constamment sur le plan spatial mais aussi dans les descriptions et les pensées d'Emma.

La liquéfication se manifeste dès la première rencontre entre Emma et Charles. Aux Bertaux, "par un temps de dégel, l'écorce des arbres suintait dans la cour, la neige sur les couvertures des bâtiments se fondait...on entendait les gouttes d'eau, une à une, tomber sur la moire tendue." (341) La moiteur reparaît dans la maison à Tostes: les murs "suintaient", les pavés sont "humides", et le bouilli fume sur le poèle. (385) Yonville n'est guère mieux: "Emma, dès le vestibule, sentit tomber sur ses épaules, comme un linge humide, le froid du plâtre." (402) et la rivière coule interminablement au fond du jardin. Donc, les espaces qu'Emma trouve isoutenables ont un lien étroit avec l'humidité.

Par contre, les espaces qui l'attirent s'associent plutôt à la vapeur. Le château de la Vaubyessard est entouré d'une brume (367). De même, quand Emma est avec Rodolphe dans la forêt, le paysage des alentours est caché dans un brouillard:

De la hauteur où ils étaient, toute la vallée paraissait un immense lac pâle, s'évaporant à l'air...les hautes lignes des peupliers, qui dépassaient la brume, figuraient des grèves que le vent remuait. (470) 
Le village d'Yonville s'évapore dans cette brume, comme dans la pensée d'Emma. La vapeur dans l'espace est donc analogue au rêve dans l'esprit: tous les deux enveloppent, cachent et transforment la réalité.

Rouen aussi est "noyée dans le brouillard" lorsqu'Emma s'y approche pour un rendez-vous avec Léon: on entend "le carillon clair des églises qui se dressaient dans la brume", et "Parfois, un coup de vent emportait les nuages vers la côte Sainte-Catherine, comme des flots aériens qui se brisaient en silence contre une falaise." (564-5) Cette dernière image résume le sort des rêves d'Emma, la falaise incarnant la dure réalité de la vie. Le brouillard est problématique: aussitôt qu'on y pénètre, il se dissipe pour dévoiler la réalité immédiate, donc il n'est efficace qu'à distance. Et n'est-il pas vrai que pour Emma: "Plus les choses, d'ailleurs, étaient voisines, plus sa pensée s'en détournait."? (379) En effet. Rouen devient de plus en plus visible tant qu'Emma s'y approche. ${ }^{8}$

Emma a des "airs évaporés" (439) et sa belle-mère s'évertue à corriger en elle "ces vapeurs-là" (440); ses rêves sont comme des nuages qui l'enveloppent. Aux Comices avec Rodolphe, par exemple, elle voit l'Hirondelle et se lance dans la rêverie; l'espace autour d'elle subit une transformation:

C'était dans cette voiture jaune que Léon, si souvent, était revenu vers elle; et par cette route là-bas qu'il était parti pour toujours! Elle crut le voir en face, à sa fenêtre, puis tout se confondit, des nuages passèrent; il lui sembla qu'elle tournait encore dans la valse, sous le feu des lustres, au bras du vicomte, et que Léon n'était pas loin, qu'il allait venir...et cependant elle sentait toujours la tête de Rodolphe à côté d'elle. (459)

Encore une fois, les nuages introduisent le rêve et obscurcissent l'espace réel.

La moiteur liée à l'espace et aux rêves informe aussi les descriptions des amours d'Emma, mais dans ce cas, les vapeurs du rêve sont reléguées par une substance plus concrète et plus réelle: l'eau. Symboliquement, l'amour dans la dimension réelle n'atteint jamais le niveau onirique des vapeurs. En effet, J-P Richard compare l'amour flaubertien a une noyade."

Rodolphe dit à Emma qu'ils sont "deux fleuves qui coulent pour se rejoindre. [leurs] pentes particulières [les] avaient poussés l'un vers l'autre." (461) Après s'être donnée à cet homme, près d'un petit étang, Emma ressent "le sang circuler dans sa chair comme un fleuve de lait." (472) Ils commencent à se rencontrer au fond du jardin de Bovary, près de la petite rivière, c'est là où l'amour prend une allure moins positive: "leur grand amour, où elle vivait plongée, parut se diminuer sous elle, comme l'eau d'un fleuve qui s'absorberait dans son lit...." (481) Quand ils se renouent, "toute leur rancune se fondit comme une neige sous la chaleur de ce baiser. “ (495) Rodolphe pense cyniquement à l'amour d'Emma: "une béatitude qui l'engourdissait; et son âme s'enfonçait en cette ivresse et s'y noyait...." (500) Quand Emma imagine une fuite avec Rodolphe, "les jours, tous magnifiques, se ressemblaient comme des flots...." (505) Dans ce cas, la vapeur du rêve est remplacée par une image de l'eau, soit parce qu'Emma est certaine que la fuite aura lieu en réalité, soit parce qu'elle reconnaît inconsciemment la véritable médiocrité de l'existence avec Rodolphe.

Pareillement, quand Léon serre la main d'Emma, "la substance même de tout 
son être lui semblait descendre dans cette paume humide." (434) Lors de leur rencontre dans la cathédrale de Rouen, Léon se hâte de consommer l'amour: "il lui semblait que son amour, qui, depuis deux heures bientôt, s'était immobilisé dans l'église, comme les pierres, allait maintenant s'évaporer telle qu'une fumée...." (547) Rodolphe a pris Emma près d'un étang, Léon la prend près de la Seine, dans une voiture qui est "ballotée comme un navire." (549)

Donc, la liquidité spatiale fonctionne comme miroir et arrière-plan pour la grande dissolution au niveau humain. L'amour peut s'évaporer dans le rêve ou couler comme l'eau vers la mort. Le dénouement réunit les deux systèmes de liquéfication (spatiale et humaine): la liquidation financière enlève l'espace domestique et déclenche une liquidation émotionnelle et physique. Quand Emma va chez Léon pour solliciter son aide, "Elle but en arrivant un grand verre d'eau." (596); rentrée chez elle, "un engourdissement la saisit. " (603) Accablée par ses malheurs et "emportée dans ses souvenirs, comme dans un torrent qui bouillonne, elle arriva bientôt à se rappeler la journée de la veille." (606) Son esprit commence à osciller, à devenir fluide.

Elle va à la Huchette; Rodolphe la trouve "ravissante à voir, avec son regard où tremblait une larme, comme l'eau d'un orage dans un calice bleu." (609) mais il refuse de l'aider. Emma s'enfuit, et la terre mouillée des champs semble l'avaler, fondre sous ses pas. Elle n'a "plus conscience d'elle-même que par le battement de ses artères...Le sol, sous ses pieds, était plus mou qu'une onde, et les sillons lui parurent d'immenses vagues brunes, qui déferlaient." (611) Elle court chez le pharmacien et demande l'arsenic d'une voix "dissolvante". (612)

Après sa mort, la dissolution et la fluidité continuent: des liquides noirs coulent de la bouche d'Emma et, dans sa chambre, Homais et Bournisien écoutent la rivière qui coule dans les ténèbres. Pendant l'enterrement, "la terre rouge, rejetée sur les bords, coulait par les coins sans bruit, continuellement." dans la fosse (634). Chaque nuit, Charles rêve d'Emma:"mais, quand il venait à l'étreindre, elle tombait en pourriture dans ses bras." (641) et juste avant de mourir lui-même il suffoque "sous les vagues effluves amoureux qui gonflaient son coeur chagrin." (645) En outre, l'espace familial est dilué par la mort: de Charles, du père Rouault, et de la mère de Charles. Berthe est orphéline sans foyer; elle est envoyée travailler dans une filature de coton. La liquidation d'Emma influence l'espace autour d'elle; en fait, dans l'oeuvre flaubertienne, "La vie ne déferle et n'écume plus, elle coule obstinément et mollement." 10

En somme, l'espace forme une union cohésive et complémentaire avec le récit dans Madame Bovary. Chez Flaubert, aucun détail n'est "gratuit" -ne dit-il pas lui-même: "Il n'y a point dans mon livre une description isolée, gratuite; toutes servent à mes personnages et ont une influence sur l'action." " -et la structure spatiale joue plusieurs rôles importants dans l'histoire: c'est une réflexion de l'état d'âme de l'héroïne, de ses rêves et de ses désillusions. En outre, c'est un moyen d'encadrer et de définir chaque personnage. Même la position et le mouvement des personnages dans l'espace sont significatifs, et le parallèlisme entre les fluidités spatiale et humaine ouvre une perspective symbolique. L'espace, beaucoup plus nuancé qu'il ne paraît, est un élément intégral de l'art de Flaubert. 


\section{Notes}

' A. Cook, "Flaubert and the Riches of Detachment,"French Review, xxxii, 2 (1958): 122. "Every detail of his narrative is made to do double duty, as observed detail and as correlative of the action."

${ }^{2}$ Gustave Flaubert, Oeuvres, vol I (Paris: Gallimard (Pléiade), 1951): 388. (Toutes les références entre parenthèses à cet ouvrage renvoient à cette édition).

${ }^{3}$ G. Poulet, "La pensée ciculaire de Flaubert," dans Debray-Genette (éd), Flaubert, miroir de la critique, (Paris: Didier, 1970): 108.

${ }^{4}$ M. Bal, Narratologie: Les Instances du Récit, (Paris: Klincksieck, 1970): 107.

${ }^{5}$ cf J. Rousset, "Madame Bovary or the book about nothing," dans R. Giraud (éd), Flaubert: A Collection of Critical Essays, (Englewood Cliffs, N.J.: Prentice-Hall, 1964): 125 "the entrance into passion is marked by an ascension above the habitual level of existence, the site of which is swallowed up and annulled beneath Emma's eyes."

'E. Auerbach, "Madame Bovary", dans Debray-Genette (éd), Flaubert, Miroir de la Critique 89.

${ }^{7}$ V. Brombert, The Novels of Flaubert, (Princeton, N.J.: Princeton University Press, 1966): 66 "Life, in the Flaubertian context, is a steady process of decay."

${ }^{8} \mathrm{cf}$ M. Bal 94-109.

'J-P Richard, "Flaubert", dans Giraud (éd) 40. "Love is a semiconscious and deliciously progressive act of drowning."

${ }^{10}$ E. Auerbach, "Madame Bovary", dans Debray-Genette (éd) Flaubert, Miroir de la Critique: 91.

" cité dans M. Bal: 92. 


\section{Ouvrages cités}

Bal, Mieke. Narratologie: les Instances du Récit. Paris: Klincksieck, 1977. Brombert, Victor. The Novels of Flaubert: A Study of Themes and

Techniques.Princeton, N.J.: Princeton University Press, 1966.

Cook, A. "Flaubert and the Riches of Detachment." French Review xxxii, 2 (déc 1958): 120-129.

Danger, Pierre. Sensations et Objets dans le Roman de Flaubert.

Paris: Colin, 1973.

Debray-Genette, Raymonde (éd). Travail de Flaubert. Paris: Seuil, 1983.

- Flaubert, Miroir de la Critique. Paris: Didier, 1970.

Demorest, D.L.L'expression Figurée et Symbolique dans L'oeuvre de Gustave Flaubert. Genève: Slatkine, 1967.

Flaubert, Gustave. Oeuvres. vol I, Paris: Gallimard (Pléiade), 1951 .

Giraud, Raymond (éd).Flaubert: A Collection of Critical Essays.

Englewood Cliffs, N.J.: Prentice-Hall, 1964.

Gothot-Mersch, Claudine. La genèse de Madame Bovary. Paris:Corti, 1966.

Sherrington, R.J. Three Novels by Flaubert: A Study of Techniques.

Oxford: Clarendon Press, 1970.

Steegmuller, Francis. Flaubert and Madame Bovary: A Double

Portrait. New York: Farrar, Straus and Company, 1950.

Thibaudet, Albert.Gustave Flaubert. Paris: Gallimard, 1935.

Vargas-Llosa, M.The perpetual orgy: Flaubert and Madame Bovary.

New York: Farrar, Straus and Giroux, 1986. 